ANL-HEP-PR-09-93, EFI-09-24

\title{
Next-to-Leading Order Cross Sections for New Heavy Fermion Production at Hadron Colliders
}

\author{
Edmond L. Bergen* \\ High Energy Physics Division, Argonne National Laboratory, Argonne, Illinois 60439, USA \\ Qing-Hong Cadt \\ High Energy Physics Division, Argonne National \\ Laboratory, Argonne, Illinois 60439, USA and \\ Enrico Fermi Institute, University of Chicago, Chicago, Illinois 60637, USA
}

\begin{abstract}
We evaluate the cross sections for new heavy fermion production at three Large Hadron Collider energies accurate to next-to-leading order in perturbative quantum chromodynamics. We treat the cases of pair production of heavy quarks via strong interactions, single heavy quark production via electroweak interactions, and the production of heavy leptons. Theoretical uncertainties associated with the choice of the renormalization scale and the parton distribution functions are specified. We derive a simple and useful parametrization of our results which should facilitate phenomenological studies of new physics models that predict new heavy quarks and/or leptons.

PACS numbers: 12.38.Bx, 12.38.Qk, 13.85.Ni, 14.65.Jk
\end{abstract}

*Electronic address: berger@anl.gov

$\dagger$ Electronic address: caoq@hep.anl.gov 


\section{INTRODUCTION}

Although the standard model (SM) of particle physics consists of three fermion generations, the number of these generations is not fixed by theory. Asymptotic freedom in quantum chromodynamics (QCD) limits the number of generations to fewer than nine. Neutrino counting, based on data at the intermediate vector boson $Z$, shows that the number of generations having light neutrinos $\left(m_{\nu} \ll m_{Z} / 2\right)$ is equal to 3 . However, neutrino oscillations suggest a new mass scale that is beyond that of the SM, and the possibility of additional heavier neutrinos is open. In addition, it has recently been emphasized that the electroweak

oblique parameters do not exclude a fourth generation of chiral fermions [1 8]. In the era of the Large Hadron Collider (LHC), the search for heavy quarks and leptons beyond those of the SM should be kept in mind.

Observation of new heavy fermions requires knowledge of their expected production cross sections and decay properties. In this work, we present inclusive cross sections of fourth generation fermion production at three LHC energies, either in pairs or singly, calculated to next-to-leading (NLO) order accuracy in QCD. Leading order Feynman diagrams for new heavy fermion production are shown for illustrative purposes in Fig. 1. We do not show the full set of NLO diagrams used in our calculations. Theoretical uncertainties associated with higher-order perturbative contributions and with the choice of parton distribution functions (PDFs) are specified. We show that the cross sections can be fitted with a rather simple analytic formula, and we present the functional form and values of the parameters for the

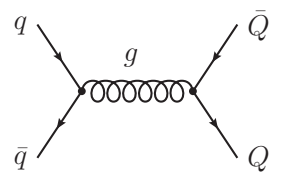

(a)

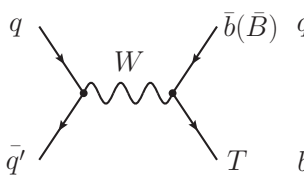

(b)

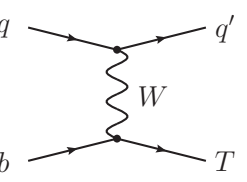

(c)

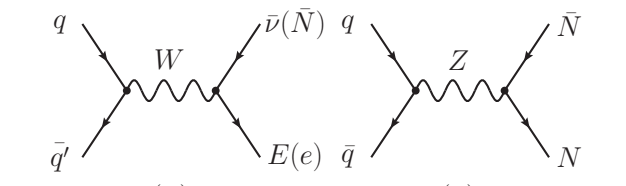

$(d)$

$(e)$

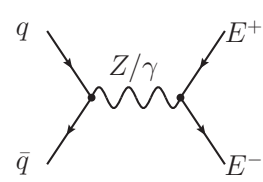

$(f)$

FIG. 1: Representative leading order Feynman diagrams for new heavy fermion production. Uppercase (lowercase) letters denote new heavy (SM) fermions. The usual gauge bosons are denoted by $g, W, Z, \gamma$. 
heavy fermion production cross sections, and their dependence on the mass of the heavy fermion.

\section{HEAVY QUARK PAIR PRODUCTION IN QCD}

Heavy quarks that carry the color charge exist in many models of new physics (NP). Examples include models with a sequential fourth generation [2, 9], or with a vectorlike $t$ prime $(T)$ [10, 11], or with a $b$ prime $(B)$ [12], or with a heavy top quark partner [13 16]. According to their physics motivation, the NP models can be further categorized into two classes. In the first group, heavy quarks are introduced to explain discrepancies between data and SM predictions. In the second, heavy quarks are essential to solve theoretical problems. For example, in Little Higgs models [13], the heavy top partner cancels the quadratic divergence in the quantum corrections to the Higgs boson mass associated with the SM top quark loop. The phenomenology of heavy quark identification at the LHC has been studied extensively $[17-25]$.

Similar to the top quark in the SM, heavy quark $Q$ can be pair produced via the strong interaction processes,

$$
q \bar{q} \rightarrow g \rightarrow Q \bar{Q} \text { and } g g \rightarrow Q \bar{Q}
$$

where $g$ denotes a gluon. In this work we assume the coupling $g-Q-\bar{Q}$ is the same as the gluon-quark interaction in the SM. The cross section for a different coupling strength can be obtained easily from our results by rescaling. In Fig. 2(a) we plot our calculated NLO inclusive cross sections for $Q \bar{Q}$ production at the LHC for three choices of the proton-proton center mass (c.m.) energy. ${ }^{1}$ The square symbol denotes the results of our exact NLO calculation whereas the curve is drawn from the simple fitting functions discussed below. As shown by the ratios presented in Fig. 2(b), an increase in the c.m. energy from 7 to $14 \mathrm{TeV}$ can enhance the total cross section markedly, depending on the value of $m_{Q}$. The values of the standard model parameters used in our calculations are found in Appendix A.

There are uncertainties in the predicted NLO cross sections associated with the choices of the renormalization scale $\left(\mu_{R}\right)$ and the factorization scale $\left(\mu_{F}\right)$ and with the choice of the PDFs. We elect to use the CTEQ6.6M PDFs [30] for our evaluations. Other sets of

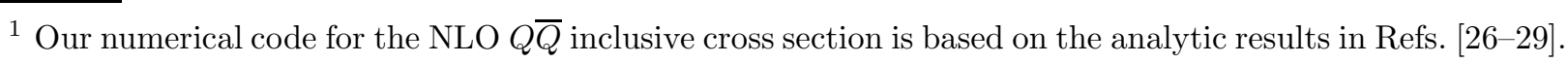



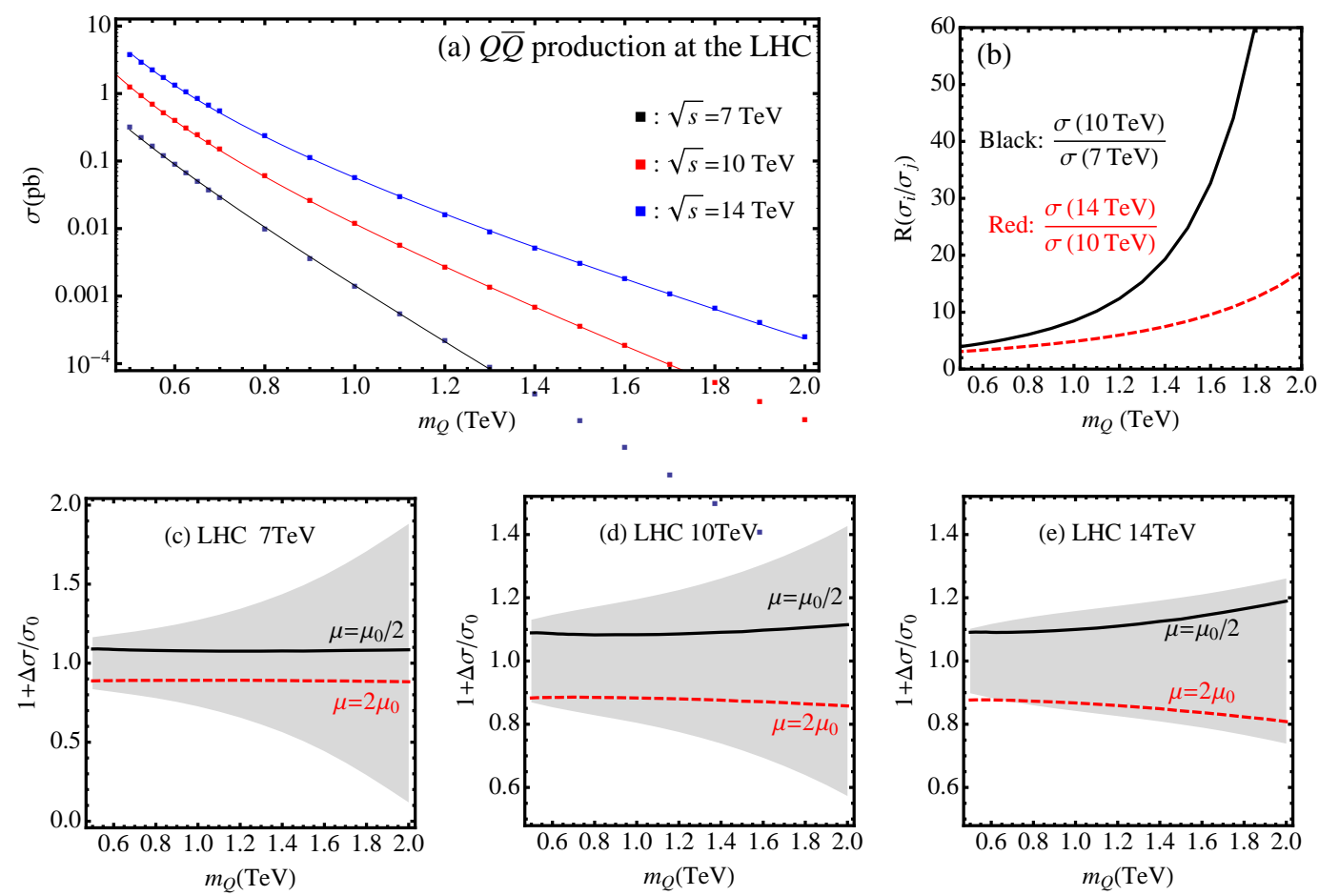

FIG. 2: (a) NLO $Q \bar{Q}$ pair production cross sections as a function of the quark mass $m_{Q}$ at the LHC. The square symbols denote the results of the exact calculation, while the curves present results of our phenomenological fit. (b) Ratios of the cross sections at different c.m. energies. (c-e) Theoretical uncertainties of the $Q \bar{Q}$ production cross section. The band denotes the PDF uncertainties, while the black solid (red dashed) curve denotes the scale dependence obtained by varying the renormalization scale by a factor 2 about the central value $\mu_{0}=m_{Q}$.

PDFs are available, notably the MSTW [31] and NNPDF [32] sets, and one might use these sets in addition to the CTEQ6.6M set to compute a range of predictions. All of these PDF sets include an analysis of the uncertainties in the PDF determinations associated both with uncertainties in the data used in the global fits and with the choices of theoretical expressions used to fit the data. A comparison may be found in Ref. [31]. In this paper, we compute the PDF uncertainty (at 90\% C.L.) from the master formula in Eq. (2.5) in Ref. [30], using all 44 sets of the CTEQ6.6M package. For heavy quark production at the large masses we are considering, the PDF uncertainties arise primarily from the valence quark PDF where different PDF sets tend to agree better than they do for gluon distributions.

In Fig. 2(c)-2(e) we display the uncertainties associated with the choices of PDFs and the 
renormalization/factorization scale. ${ }^{2}$ The uncertainties are portrayed relative to the cross section with the best-fit PDF by the bands in Fig. 2, The PDF uncertainties are relatively large because the mass of the heavy quark requires that the PDFs be sampled in regions where they are relatively unconstrained. The PDF uncertainties decrease with increasing c.m. energy.

The uncertainties in the NLO cross section associated with the renormalization scale $\left(\mu_{R}\right)$ and factorization scale $\left(\mu_{F}\right)$ are shown in Fig. 2. These uncertainties can be considered as an estimate of the size of unknown higher-order contributions. In this study, we set $\mu=\mu_{R}=\mu_{F}$ and vary it around the central value of $\mu_{0}=m_{Q}$, where $m_{Q}$ is the mass of the heavy quark. Typically, a factor of 2 is used as a rule of thumb, and we display curves with $\mu=2 \mu_{0}$ and $\mu=\mu_{0} / 2$. In Figs. 2(c)-2(e) we plot $1+\sigma\left(\mu_{i}\right) / \sigma\left(\mu_{0}\right)$ as a function of $m_{Q}$. The cross sections vary between about $-10 \%$ for $\mu=2 \mu_{0}$ and $+10 \% \sim 15 \%$ for $\mu=\mu_{0} / 2$. We note that the scale dependence at the three energies is insensitive to $m_{Q}$. It is comparable to the PDF uncertainties in the region of relatively small $m_{Q}$ but is much smaller than the PDF uncertainties in the region of large $m_{Q}$.

We find that our calculated cross sections can be fitted well by a simple three parameter analytic expression:

$$
\log \left[\frac{\sigma\left(m_{Q}, \mu\right)}{\mathrm{pb}}\right]=A(\mu)\left(\frac{m_{Q}}{\mathrm{TeV}}\right)^{-1}+B(\mu)+C(\mu)\left(\frac{m_{Q}}{\mathrm{TeV}}\right),
$$

where the units of $\sigma$ and $m_{Q}$ are picobarn $(\mathrm{pb})$ and $\mathrm{TeV}$. The variation with respect to the reference cross section (corresponding to $\mu=m_{Q}$ ) gives the NLO scale dependence $\Delta_{\mu}\left(m_{Q}\right)$. The values of $\Delta_{\mu}$, and coefficients $A, B, C$ are listed in Table I. Our results are presented in the form

$$
\sigma=\sigma\left(\mu_{0}\right)_{\Delta \sigma\left(2 \mu_{0}\right)}^{\Delta \sigma\left(\mu_{0} / 2\right)}
$$

where $\sigma\left(\mu_{0}\right)$ is our prediction at the scale $\mu_{0}$. The cross sections at the scale $\mu_{0} / 2$ and $2 \mu_{0}$ read as $\sigma\left(\mu_{0} / 2\right)=\sigma\left(\mu_{0}\right)+\Delta \sigma\left(\mu_{0} / 2\right)$ and $\sigma\left(2 \mu_{0}\right)=\sigma\left(\mu_{0}\right)+\Delta \sigma\left(2 \mu_{0}\right)$, respectively.

As is evident in Fig. 2(a), the fitting function and parameters in Table I provide an excellent representation of the calculated cross sections over the range of heavy quark masses

2 For the scale dependence, $\Delta \sigma / \sigma_{0}$ is defined as $\left(\sigma\left(\mu_{i}\right)-\sigma\left(\mu_{0}\right)\right) / \sigma\left(\mu_{0}\right)$, while for the PDF dependence $\Delta \sigma$ is defined as $\Delta \sigma=1 / 4 \sum_{i}^{n}\left(\sigma_{i}^{+}-\sigma_{i}^{-}\right)^{2}$, where where $\sigma_{i}^{ \pm}$denotes the upper (lower) cross section for the $i$ th eigenvector of the PDF sets and $n=22$ for CTEQ6.6M PDF sets. The PDF uncertainties are then plotted as $1 \pm \Delta \sigma / \sigma_{0}$ where $\sigma_{0}$ stands for the cross section of the averaged PDF set. This definition applies to all other figures with cross section uncertainties. 


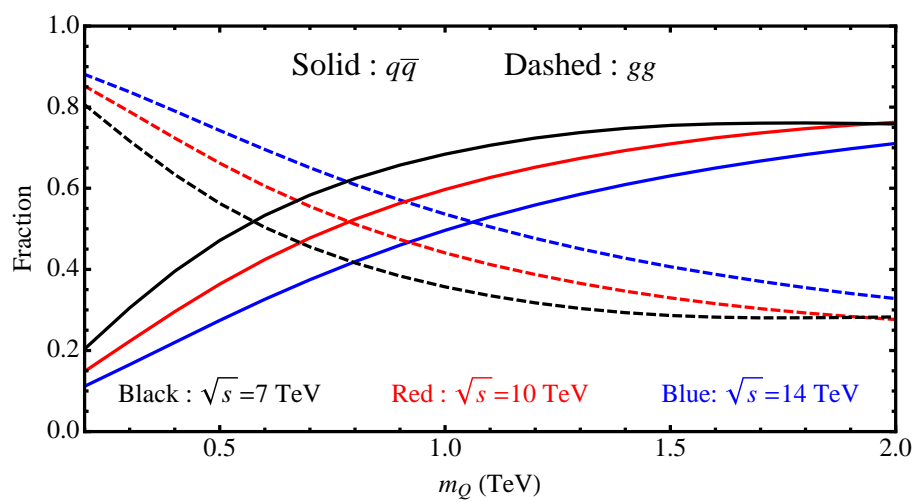

FIG. 3: Fractions of the $q \bar{q}$ and $g g$ initial state contributions to the NLO total cross sections for $Q \bar{Q}$ pair production at the LHC.

shown in the figure. We caution that the expression should not be used outside this range. One could extend the region of applicability of a fit to lower mass, say down to the top quark mass, by simply increasing the number of terms in the polynomial expression. In Appendix B we extend our fit of the cross sections for heavy quark pair production to cover the wider mass range, 250 to $700 \mathrm{GeV}$. We also present a tabulation showing our exact NLO calculation and our fitted results. The numerical comparison shows that our fitted values agree with the exact calculations well within $1 \%$. Interested readers may contact the authors for accurate fitting functions that cover the mass range from the top quark mass to $4 \mathrm{TeV}$.

One reason more terms may be needed in a polynomial expression is that processes with initial state gluons play an important role in pair production of relatively light quarks. As shown in Fig. 3, at $\sqrt{s}=7 \mathrm{TeV}$, the gluon initial state contribution dominates over the quark initial state when $m_{Q}<550 \mathrm{GeV}$; at $\sqrt{s}=10 \mathrm{TeV}$ when $m_{Q}<750 \mathrm{GeV}$; while at $\sqrt{s}=14 \mathrm{TeV}$ when $m_{Q}<1 \mathrm{TeV}$. For relatively light quark production, say $m_{Q}<300 \mathrm{GeV}$, $1 / y^{2}$ and $1 / y^{3}$ terms $\left(y \equiv m_{Q} / \mathrm{TeV}\right)$ are required to fit the total cross section. In Appendix $\mathrm{C}$, we explore the apparent process independence of the remarkably simple analytic expression Eq. 1 and discuss its range of applicability in the heavy fermion mass.

In view of the interest in the top quark as the heaviest of the known quarks, we provide NLO predictions of the top quark pair production cross sections in Table II at three LHC energies for a few values of $m_{t}$. Our result at $14 \mathrm{TeV}$ is consistent with Ref. [33]. 
TABLE I: Fitting parameters $(A, B, C)$ of the parametric formula [Eq. [1] for the NLO total cross sections $(\mathrm{pb})$ for heavy quark production at the LHC. The central values are given for the scale choice $\mu_{R}=\mu_{F}=\mu_{0}=m_{Q}$. The superscript and subscript denote the deviations in the coefficients from the central value when the scale is $\mu_{0} / 2$ (superscript) or $2 \mu_{0}$ (subscript). We use the CTEQ6.6M PDFs.

\begin{tabular}{|c|c|c|c|c|}
\hline Process & Parameter & $\sqrt{s}=7 \mathrm{TeV}$ & $\sqrt{s}=10 \mathrm{TeV}$ & $\sqrt{s}=14 \mathrm{TeV}$ \\
\hline \multirow{3}{*}{$q q / g g \rightarrow Q \bar{Q}$} & $A$ & $0.84569_{-0.03441}^{+0.04779}$ & $1.76661_{-0.01912}^{+0.03079}$ & $2.03833_{-0.01292}^{+0.02345}$ \\
\hline & $B$ & $1.48655_{-0.02244}^{-0.05339}$ & $-0.39194_{-0.06751}^{+0.00546}$ & $-0.45930_{-0.08482}^{+0.03295}$ \\
\hline & $C$ & $-8.87048_{-0.08488}^{+0.09955}$ & $-5.82142_{-0.03726}^{+0.04314}$ & $-4.45853_{-0.01642}^{+0.01784}$ \\
\hline \multirow{3}{*}{$q b \rightarrow q^{\prime} T$} & $A$ & $0.72823_{-0.01262}^{+0.00130}$ & $0.72901_{+0.00981}^{-0.00419}$ & $0.69166_{+0.01911}^{-0.00600}$ \\
\hline & $B$ & $1.66318_{+0.00630}^{-0.03563}$ & $2.28354_{+0.00541}^{-0.01591}$ & $2.94279_{-0.01098}^{-0.01395}$ \\
\hline & $C$ & $-3.38334_{-0.04100}^{+0.05251}$ & $-2.57503_{-0.03535}^{+0.03980}$ & $-2.06090_{-0.02618}^{+0.03918}$ \\
\hline \multirow{3}{*}{$q \bar{b} \rightarrow q^{\prime} \bar{T}$} & $A$ & $0.77742_{-0.00158}^{+0.00580}$ & $0.78617_{+0.00520}^{+0.00133}$ & $0.75829_{+0.00743}^{-0.00266}$ \\
\hline & $B$ & $0.86229_{+0.03096}^{-0.04447}$ & $1.53387_{+0.01617}^{-0.03343}$ & $2.22983_{+0.01447}^{-0.01720}$ \\
\hline & $C$ & $-3.57353_{-0.05169}^{+0.05666}$ & $-2.72791_{-0.03900}^{+0.04759}$ & $-2.18287_{-0.03617}^{+0.03754}$ \\
\hline \multirow{3}{*}{$q \overline{q^{\prime}} \rightarrow T \bar{b}$} & $A$ & $1.37193_{+0.01703}^{-0.00541}$ & $1.41641_{+0.00749}^{+0.00152}$ & $1.46925_{+0.00596}^{-0.00453}$ \\
\hline & $B$ & $-3.44671_{-0.05685}^{+0.03710}$ & $-3.45805_{-0.02949}^{+0.01625}$ & $-3.33539_{-0.02316}^{+0.02351}$ \\
\hline & $C$ & $-5.22956_{-0.01643}^{+0.02415}$ & $-3.81301_{-0.01891}^{+0.02269}$ & $-3.00593_{-0.01352}^{+0.01228}$ \\
\hline \multirow{3}{*}{$q \overline{q^{\prime}} \rightarrow \bar{T} b$} & $A$ & $1.79403_{+0.02359}^{-0.00423}$ & $1.77007_{+0.00967}^{-0.00699}$ & $1.68940_{+0.00815}^{-0.00650}$ \\
\hline & $B$ & $-5.40590_{-0.06414}^{+0.02628}$ & $-5.03094_{-0.03479}^{+0.03140}$ & $-4.44604_{-0.02747}^{+0.02719}$ \\
\hline & $C$ & $-4.81694_{-0.01401}^{+0.02980}$ & $-3.67831_{-0.01460}^{+0.01489}$ & $-3.08803_{-0.01088}^{+0.00993}$ \\
\hline \multirow{3}{*}{$q \overline{q^{\prime}} \rightarrow T \bar{B}$} & $A$ & $0.09505_{+0.00202}^{-0.00087}$ & $0.98804_{-0.00090}^{+0.01201}$ & $1.07010_{+0.00304}^{-0.00263}$ \\
\hline & $B$ & $-1.08915_{-0.03419}^{+0.03084}$ & $-2.21256_{-0.00969}^{-0.01419}$ & $-2.20446_{-0.01452}^{+0.01778}$ \\
\hline & $C$ & $-10.4782_{-0.04719}^{+0.04744}$ & $-4.66219_{-0.03293}^{+0.04337}$ & $-3.75217_{-0.01956}^{+0.01638}$ \\
\hline \multirow{3}{*}{$q \overline{q^{\prime}} \rightarrow \bar{T} B$} & $A$ & $0.65738_{-0.00549}^{+0.01149}$ & $1.15500_{+0.00280}^{-0.00182}$ & $1.18663_{+0.00487}^{-0.00354}$ \\
\hline & $B$ & $-4.02175_{-0.00550}^{-0.01027}$ & $-5.09831_{-0.02683}^{+0.02561}$ & $-4.73348_{-0.02800}^{+0.02658}$ \\
\hline & $C$ & $-9.05880_{-0.06865}^{+0.07512}$ & $-6.08544_{-0.03100}^{+0.02944}$ & $-4.91903_{-0.01835}^{+0.01753}$ \\
\hline
\end{tabular}


TABLE II: NLO top quark pair production cross sections (pb) at the LHC with three different c.m. energies based on the CTEQ6.6M PDFs. The central values are given for the scale choice $\mu_{R}=\mu_{F}=\mu_{0}=m_{t}$. The superscript and subscript denote the deviations of the coefficients from the central value when the scale is $\mu_{0} / 2$ (superscript) or $2 \mu_{0}$ (subscript).

\begin{tabular}{c|ccc}
\hline$m_{t}(\mathrm{GeV})$ & $\sqrt{s}=7 \mathrm{TeV}$ & $\sqrt{s}=10 \mathrm{TeV}$ & $\sqrt{s}=14 \mathrm{TeV}$ \\
\hline 171 & $157.4_{-20.3}^{+18.9}$ & $396.5_{-48.4}^{+47.2}$ & $877.2_{-101.4}^{+103.1}$ \\
173 & $148.2_{-19.2}^{+17.7}$ & $374.5_{-45.8}^{+44.4}$ & $830.9_{-94.1}^{+93.9}$ \\
175 & $139.6_{-18.1}^{+16.5}$ & $354.0_{-43.4}^{+41.6}$ & $787.5_{-91.5}^{+91.3}$ \\
177 & $131.6_{-17.0}^{+15.6}$ & $335.0_{-41.1}^{+39.2}$ & $747.2_{-86.9}^{+86.4}$ \\
\hline
\end{tabular}

\section{HEAVY QUARK PRODUCTION THROUGH THE ELECTROWEAK IN- TERACTION}

Heavy quark production occurs also through electroweak interactions. One example is the heavy top quark partner $(T)$ in the Little Higgs Model [14, 15], which is responsible for canceling large quantum corrections to the Higgs boson mass from the SM top quark loop. In addition to its pair production through QCD interactions, the $T$ quark can be produced singly in association with a light quark through the processes

$$
q b \rightarrow q^{\prime} T, \quad \text { and } \quad q \bar{q}^{\prime} \rightarrow T b .
$$

It may also be produced in association with a heavy $B$ quark via the $s$-channel subprocess

$$
q \bar{q}^{\prime} \rightarrow W^{*} \rightarrow T B
$$

We consider first single $T$ quark production in association with a SM $b$ quark via an $s$-channel subprocess, illustrated at leading order in Fig. 1(b). This subprocess involves a weak interaction vertex $W-T-b$ and a quark mixing matrix $V_{T b}$. For simplicity, we assume that the coupling of $W-T-b$ is same as the SM $W-t-b$ coupling and that $\left|V_{T b}\right|=1$. Our results may be rescaled for other choices. We calculate the $T \bar{b}$ and $\bar{T} b$ results separately since the $p p$ initial state at the $\mathrm{LHC}$ is not a $C P$ eigenstate. The renormalization and factorization scales are chosen to be $\mu_{R}=\mu_{F}=\sqrt{Q_{V}^{2}}$ where $Q_{V}$ denotes the four-momentum of the $W$ boson in the propagator.

The square symbols in Fig. 4 show the predicted production cross sections for $T \bar{b}$ and $\bar{T} b$ at the LHC for three c.m. energies. The curves are our fit with the parametrization in Eq. 1. 

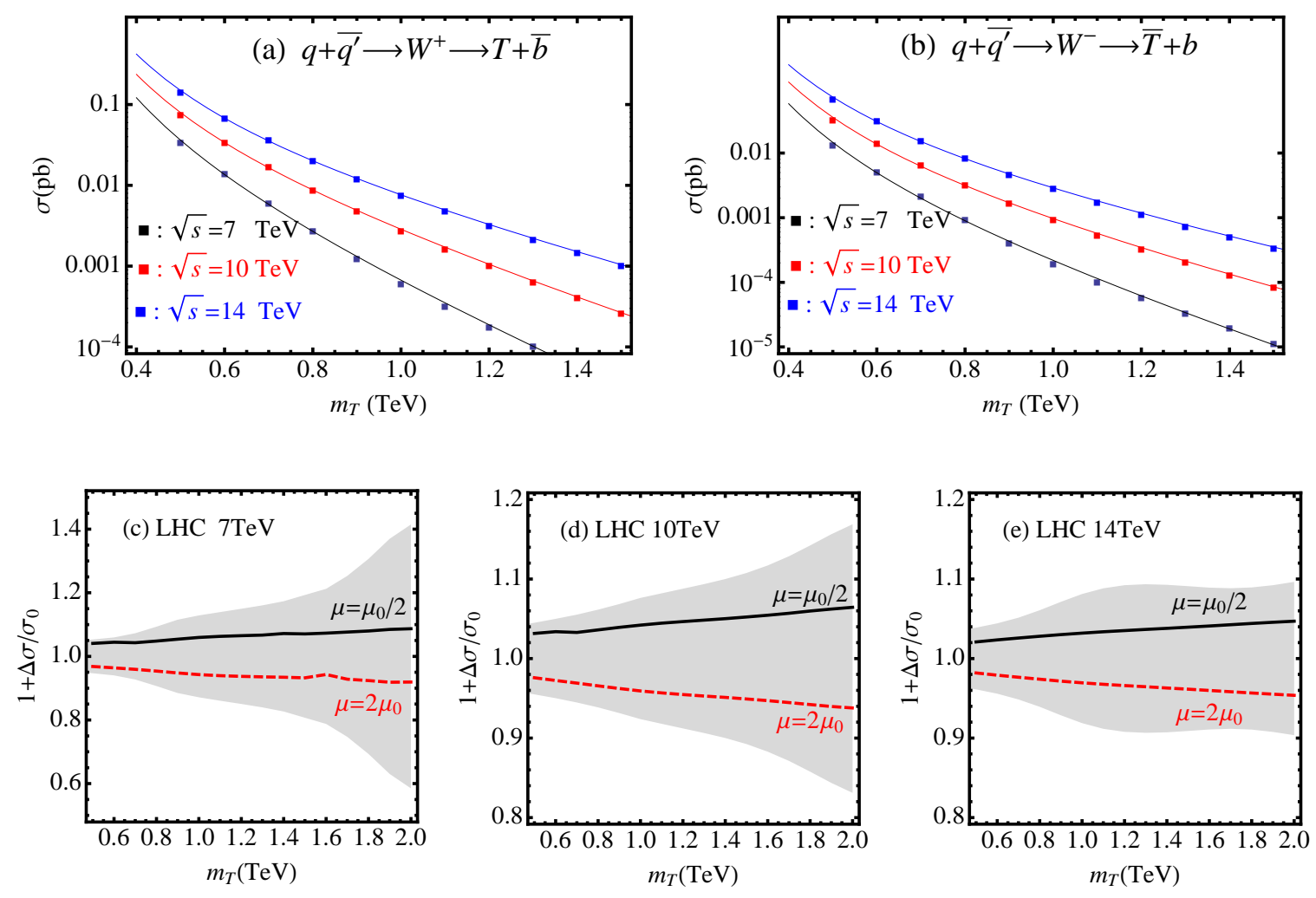

FIG. 4: NLO production cross sections for (a) $T \bar{b}$ and (b) $\bar{T} b$ at the LHC as a function of $m_{T}$, and (c-e) PDF and scale uncertainties of the $T \bar{b}$ production cross section. The $\bar{T} b$ production cross section exhibits similar uncertainties.

We note that this simple parametrization fits single $T$ production via the $s$-channel process very well for the three LHC energies. The fitting parameters are presented in Table 【. The cross section for single $T$ production is larger than the cross section for single $\bar{T}$ production by almost a factor $2 \sim 3$. This is due to the difference in parton densities of the colliding protons. While in both cases the antiquark is from the quark sea of one of the incoming protons, the probability that it collides with an up quark from the other proton is higher than the probability for a collision with a down quark. Since $T \bar{b}$ and $\bar{T} b$ production exhibit similar uncertainties, we present only the results for $T \bar{b}$ production in Figs. 4(c-e). We note the scale dependence at the three energies is not very sensitive to $m_{T}$, and it is much smaller than the PDF uncertainties in the large $m_{T}$ region. An increase in the c.m. energy reduces the PDF uncertainties in the large $m_{T}$ region sizably.

In the "sequential" fourth generation model, the heavy $T$ and $B$ quarks form an isodou- 

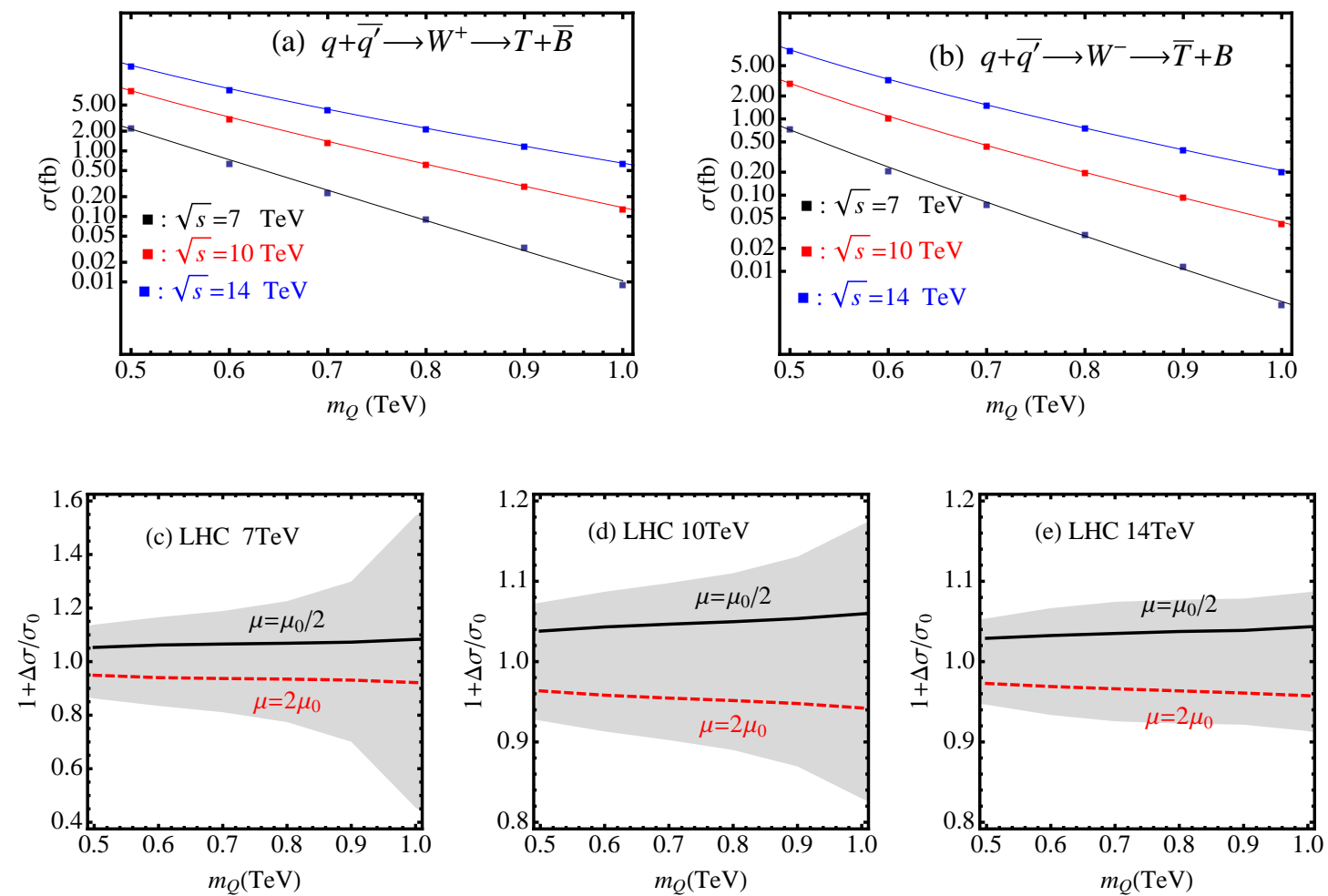

FIG. 5: NLO production cross sections for (a) $T \bar{B}$ and (b) $\bar{T} B$ at the LHC as a function of $m_{Q}=m_{T}=m_{B}$, and (c-e) PDF and scale uncertainties of the $T \bar{B}$ production cross section. The $\bar{T} B$ production cross section exhibits similar uncertainties.

blet, and their interaction with the $W$ boson is identical to the $W-t-b$ interaction in the SM with the substitution of $V_{t b} \rightarrow V_{T B}=1$. For simplicity, we take the $T$ and $B$ quarks to be degenerate, i.e. $m_{T}=m_{B}=m_{Q}$. The $T$ and $B$ quarks can be produced together via the Drell-Yan process (with an $s$-channel $W^{*}$ ). In Fig. 5 we show our calculated cross sections and the fitted curves based on the parametrization in Eq. 1. The parametrization works well at $\sqrt{s}=10 \mathrm{TeV}$ and $14 \mathrm{TeV}$ but less adequately at $7 \mathrm{TeV}$. The inclusion of more terms in the polynomial would improve the fit. The fitting parameters are listed in Table I.

Last, we consider single $T$ quark production via a $t$-channel exchange diagram, illustrated at leading order in Fig. 1(c). The production cross section is larger than in the s-channel case because it is not subject to $1 / s$ suppression. In this calculation we adopt the so-called double deep inelastic-scattering scale proposed in Ref. [34]. Color conservation enforces a natural factorization of the scales. The fermion line in Fig. 1(c) that does not include a heavy quark probes a proton with the deep inelastic-scattering scale $\sqrt{Q_{V}^{2}}$, which is identical to 

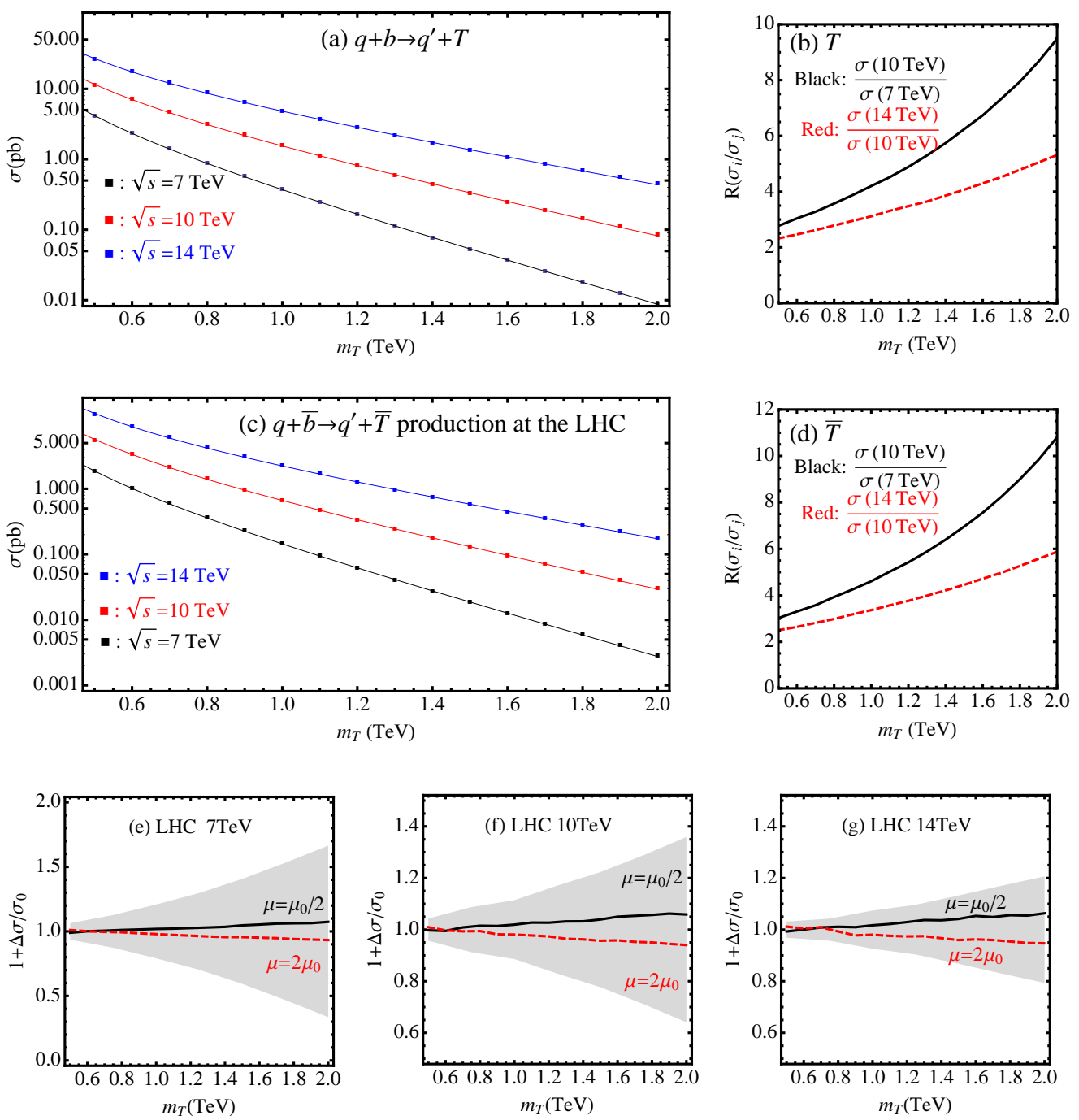

FIG. 6: Single heavy (a) $T$ production and (c) $\bar{T}$ production via the $t$-channel subprocess as a function of $m_{T}$. The square symbols denote the results of the exact calculation while the curves present results of our phenomenological fit. (b) and (d) Ratios of the cross sections at different c.m. energies. (e-g) Theoretical uncertainties of the single $T$ production cross section. The band denotes the PDF uncertainties while the black solid (red dashed) curve denotes the scale dependence obtained by varying the renormalization scale by a factor 2 about the central value $\mu_{0}=m_{T}$.

the virtuality of the $W$ boson through NLO. The fermion line that connects to a heavy quark sees the deep inelastic-scattering scale for massive quarks of $\sqrt{Q_{V}^{2}+m_{Q}^{2}}$.

Our simple parametrization also works well for the $t$-channel results as may be seen in Figs. 6(a) and 6(c). The fitting parameters are found in Table I. Again, an increase in the c.m. energy from 7 to $14 \mathrm{TeV}$ can enhance the total cross section markedly, depending 
on the value of $m_{T}$; see Figs. 6(b) and 6(d). The PDF and scale uncertainties are shown in Figs. 6(e)-6(g). Since single $T$ and single $\bar{T}$ production exhibit similar uncertainties, we present only the results for single $T$ production. We note the scale dependence at the three energies is not sensitive to $m_{T}$. The cross sections vary between $\pm 2 \%$ for $m_{T} \sim 500 \mathrm{GeV}$ to $\pm 7 \%$ for $m_{T} \sim 2 \mathrm{TeV}$. The scale dependence is much smaller than the PDF uncertainties in the large $m_{T}$ region.

\section{EXOTIC LEPTON PRODUCTION}

Several variants of the seesaw mechanism have been proposed to explain light neutrino masses. Positive signals could be observed at the LHC in the case that seesaw messengers exist at the $\mathrm{TeV}$ scale or below. Three types of tree-level seesaw models generate light neutrino Majorana masses via introduction of (1) a right-handed neutrino singlet, (2) a complex scalar triplet $\Delta$ with hypercharge $Y=1$, and (3) a lepton triplet $\Sigma$ with $Y=0$. In these seesaw models, neutrinos achieve their masses via a lepton number violating operator at the scale $\Lambda$. The scale is not necessarily very high, and it might be around a TeV. Searches for these exotic leptons and, if observed, measurement of their properties would verify and even distinguish different seesaw mechanisms [35]. For this purpose, an accurate calculation including higher-order QCD corrections is in order.

TABLE III: Coupling strengths of the gauge vertices in heavy lepton pair production for different models, where $g$ denotes the usual weak coupling strength. Note that all the couplings are vectorlike.

\begin{tabular}{lcccc}
\hline & $W E N$ & $Z E E$ & $\gamma E E$ & $Z N N$ \\
\hline Majorana triplet & $g$ & $g c_{W}$ & $e$ & $\ldots$ \\
Dirac triplet & $g$ & $g c_{W}$ & $e$ & $\ldots$ \\
Lepton isodoublet & $\frac{g}{\sqrt{2}}$ & $\frac{g}{c_{W}}\left(-\frac{1}{2}+s_{W}^{2}\right)$ & $e$ & $\frac{g}{2 c_{W}}$ \\
Charge singlet & $\ldots$ & $\ldots$ & $e$ & $\ldots$ \\
\hline
\end{tabular}


We examine the following three possibilities for exotic lepton production:

$$
\begin{aligned}
& q \bar{q} \rightarrow \gamma / Z \rightarrow E^{+} E^{-}, \\
& q \bar{q} \rightarrow Z \rightarrow N N, \\
& q \overline{q^{\prime}} \rightarrow W \rightarrow E N,
\end{aligned}
$$

where $E(N)$ denotes the new heavy charged lepton (heavy neutrino). The interactions of gauge bosons and heavy leptons are summarized in Table $\amalg$ for four interesting NP models: (1) a Majorana lepton triplet with $Y=0,(2)$ a Dirac lepton triplet with $Y=0$, (3) a lepton isodoublet, (4) a charge singlet. The motivation for these models and more details can be found in Ref. [36]. It is worth mentioning the following properties:

- All the interactions given in the Table III are vectorlike.

- There are no $Z N N$ interactions in the Majorana/Dirac lepton triplet model because the triplet has zero hypercharge, and $N N$ pairs are not produced.

- A Dirac lepton triplet is formed by two degenerate Majorana triplets with opposite CP parities. As shown in Ref. [37], the heavy lepton fields can be redefined in such a way that the Lagrangian is written in terms of two charged leptons $E_{1}^{-}, E_{2}^{+}$and a Dirac neutrino $N$. As a consequence of the presence of two charged fermions instead of only one, the total heavy lepton production cross section is twice that for a Majorana triplet. In this work, however, we consider only one flavor of charged lepton in both the Majorana triplet and the Dirac triplet.

To make our results more useful, rather than focusing on a specific NP model, we consider the following effective interaction in our numerical calculation,

$$
\mathcal{L}=-\frac{g}{c_{W}}\left(\bar{E} \gamma^{\mu} E+\bar{N} \gamma^{\mu} N\right) Z_{\mu}-\frac{g}{\sqrt{2}}\left(\bar{E} \gamma^{\mu} N W_{\mu}^{-}+\bar{N} \gamma^{\mu} E W_{\mu}^{+}\right)+e \bar{E} \gamma^{\mu} E A_{\mu},
$$

where the symbol $g$ stands for the usual weak coupling strength. Numerical results for the models listed in Table $\Pi 1$ can be easily derived from ours by simply rescaling the coupling strengths, except for the process $q \bar{q} \rightarrow \gamma / Z \rightarrow E^{+} E^{-}$where there are interference effects of the photon and $Z$ boson contributions. However, since the threshold for two heavy charged leptons is much higher than the $Z$ boson mass, the interference effects are subleading. We present separately the NLO cross sections for $q \bar{q} \rightarrow \gamma \rightarrow E^{+} E^{-}$and $q \bar{q} \rightarrow Z \rightarrow E^{+} E^{-}$; the 

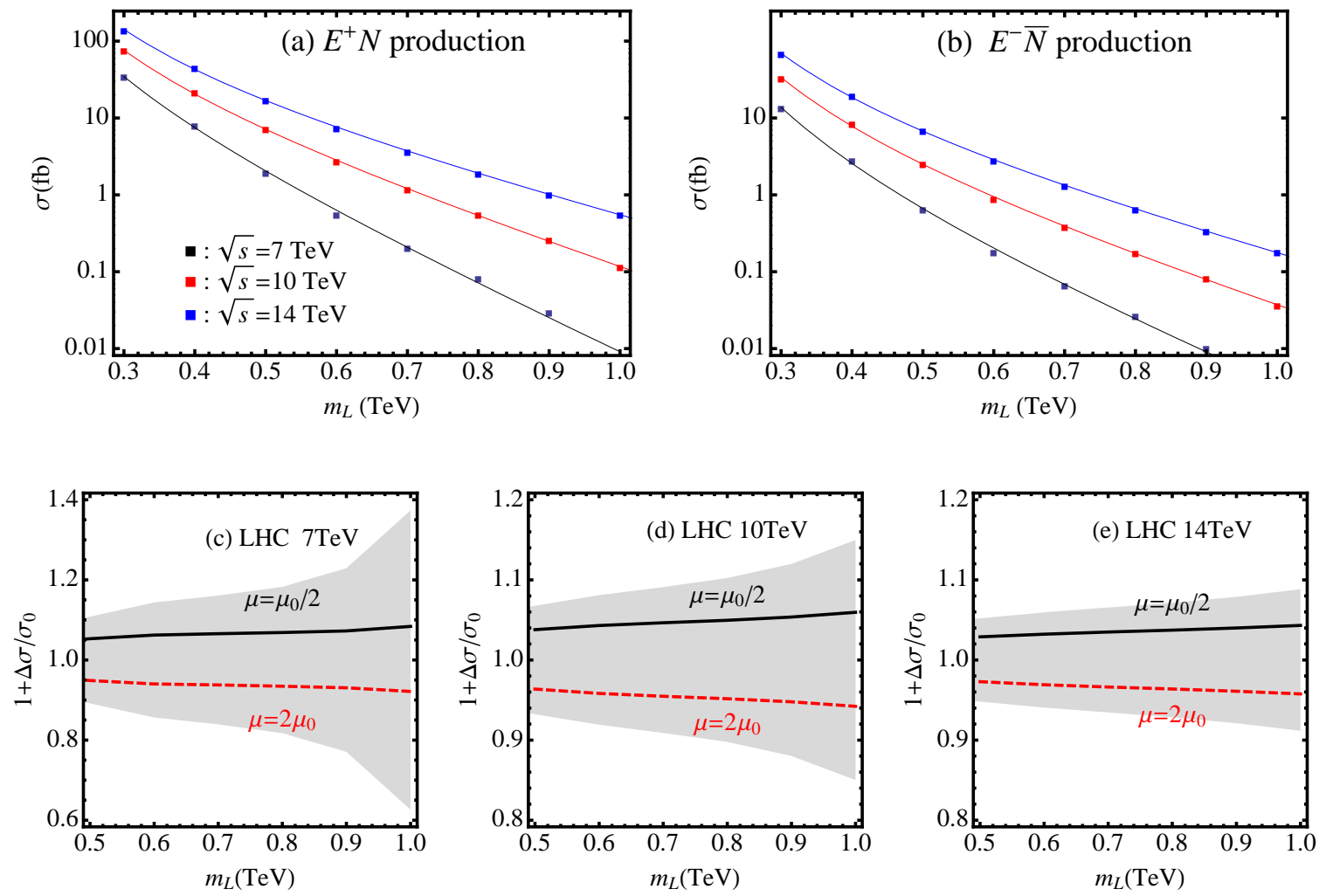

FIG. 7: NLO production cross sections for (a) $E^{+} N$ and (b) $E^{-} \bar{N}$ production at the LHC as a function of $m_{L}=m_{E}=m_{N}$, and (c-e) PDF and scale uncertainties of $E^{+} N$ production. The $E^{-} \bar{N}$ production cross section exhibits similar uncertainties.

couplings involved in the two processes are overall factors. For all the $s$-channel processes listed above, the renormalization and factorization scales are chosen to be $\mu=\sqrt{Q_{V}^{2}}$ where $Q_{V}$ denotes the four-momentum of the gauge boson in the propagator. For simplicity, we take the $E$ and $N$ leptons to be degenerate, i.e. $m_{E}=m_{N}=m_{L}$.

In Figs. 7(a) and 7(b) we display the NLO cross sections for the $q \overline{q^{\prime}} \rightarrow W^{+} \rightarrow E^{+} N$ and $q \bar{q}^{\prime} \rightarrow W^{-} \rightarrow E^{-} \bar{N}$ processes, respectively. Both processes exhibit similar scale and PDF uncertainties as shown in Figs. 7 (c)-7(e). The fitting parameters are given in Table IV.

We also plot the NLO cross sections for the $q \bar{q} \rightarrow \gamma \rightarrow E^{+} E^{-}$and the $q \bar{q} \rightarrow Z \rightarrow E^{+} E^{-}$ processes in Fig. 8. Both processes exhibit similar scale and PDF uncertainties as shown in Fig. 8 (c)-8(e). Note that the cross sections for $q \bar{q} \rightarrow Z \rightarrow \bar{N} N$ are the same as those for $q \bar{q} \rightarrow Z \rightarrow E^{+} E^{-}$owing to the same assignment of couplings in Eq. 3; therefore, they are not shown here. 

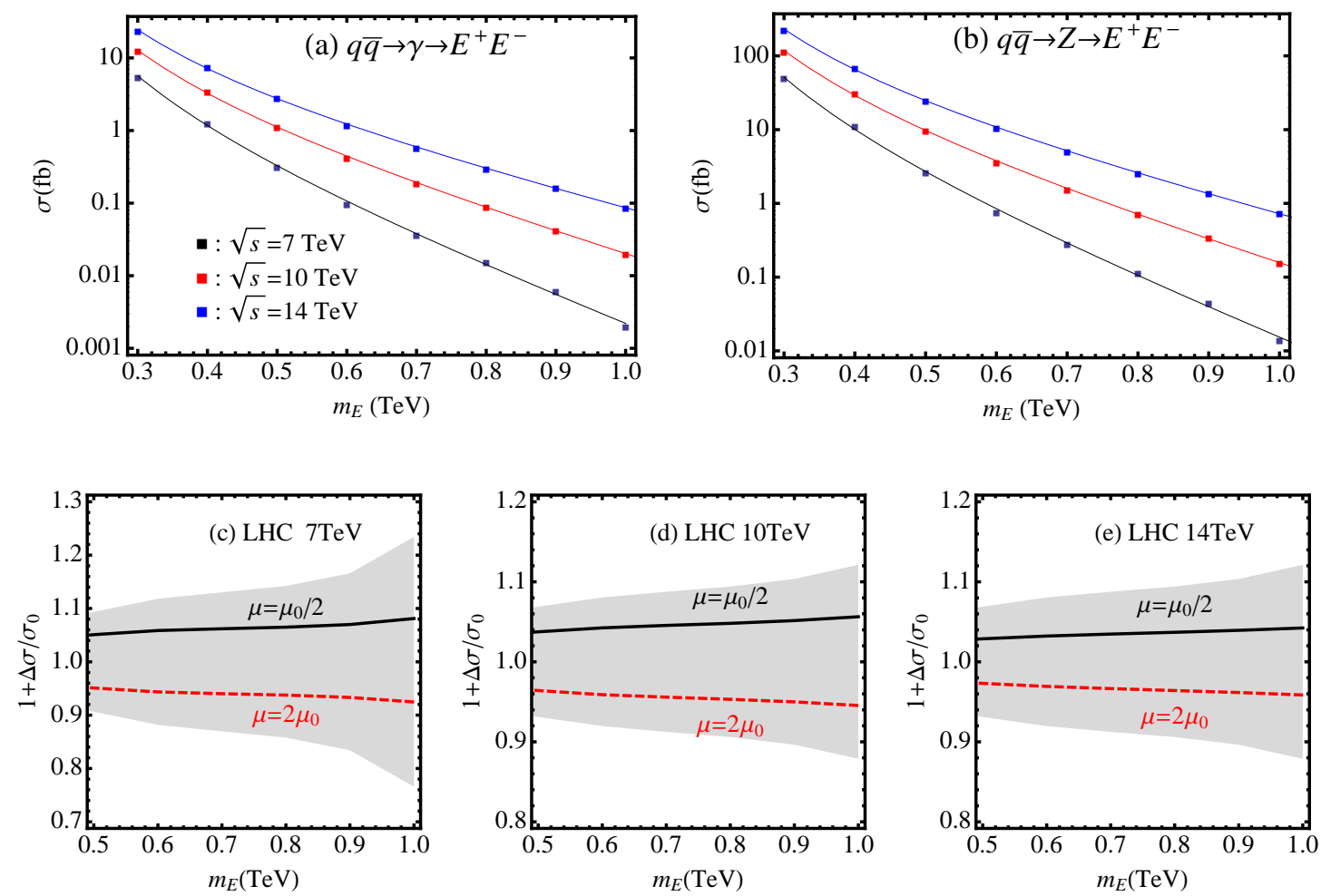

FIG. 8: NLO production cross sections for (a) $E^{+} E^{-}$production via an intermediate virtual photon and (b) via an intermediate $Z$ boson at the LHC as a function of $m_{E}$, and (c-e) PDF and scale uncertainties of $E^{+} E^{-}$production via an intermediate virtual photon. Production via an intermediate $Z$ boson exhibits similar uncertainties.

\section{SUMMARY}

We present NLO predictions for the cross sections for new heavy quark and lepton production at three Large Hadron Collider energies. We treat the cases of pair production of heavy quarks via strong interactions, single heavy quark production via electroweak interactions, and the production of exotic heavy leptons. Theoretical uncertainties associated with the choice of the renormalization scale and the parton distribution functions are specified. We derive a simple and useful parametrization of our results which should assist in experimental simulations and in phenomenological studies of new physics models containing new heavy quarks and/or leptons.

Acknowledgments. E. L. B. is supported at Argonne by the U. S. Department of Energy under Contract No. DE-AC02-06CH11357. Q. H. C. is supported in part by the Argonne 
TABLE IV: The fitting parameters $(A, B, C)$ in the parametric formula [Eq. 1] for the NLO total cross sections $(\mathrm{pb})$ for heavy lepton production at the LHC. The central values are given for the scale choice $\mu_{R}=\mu_{F}=\mu_{0}=m_{E}$. The superscript and subscript denote the deviations of the coefficients from the central value when the scale is $\mu_{0} / 2$ (superscript) or $2 \mu_{0}$ (subscript). We use the CTEQ6.6M PDFs.

\begin{tabular}{|c|c|c|c|c|}
\hline Process & Parameter & $\sqrt{s}=7 \mathrm{TeV}$ & $\sqrt{s}=10 \mathrm{TeV}$ & $\sqrt{s}=14 \mathrm{TeV}$ \\
\hline \multirow{3}{*}{$q \overline{q^{\prime}} \rightarrow E^{+} N$} & $A$ & $0.71010_{+0.00943}^{+0.00073}$ & $0.77778_{+0.00406}^{-0.00278}$ & $0.81919_{+0.00335}^{-0.00250}$ \\
\hline & $B$ & $-2.90505_{-0.05227}^{+0.02141}$ & $-3.16717_{-0.02697}^{+0.02513}$ & $-3.11747_{-0.02179}^{+0.02228}$ \\
\hline & $C$ & $-9.41140_{-0.09655}^{+0.05659}$ & $-6.66323_{-0.03572}^{+0.03454}$ & $-5.20543_{-0.02455}^{+0.02221}$ \\
\hline \multirow{3}{*}{$q \overline{q^{\prime}} \rightarrow E^{-} \bar{N}$} & $A$ & $0.99258_{+0.00593}^{-0.00287}$ & $0.98794_{+0.00560}^{-0.00431}$ & $0.94199_{+0.00486}^{-0.00381}$ \\
\hline & $B$ & $-5.03622_{-0.03759}^{+0.02662}$ & $-4.74631_{-0.03380}^{+0.03171}$ & $-4.18717_{-0.02725}^{+0.02669}$ \\
\hline & $C$ & $-8.53154_{-0.04582}^{+0.05327}$ & $-6.43970_{-0.02674}^{+0.02594}$ & $-5.39397_{-0.01906}^{+0.01772}$ \\
\hline \multirow{3}{*}{$q \bar{q} \rightarrow \gamma \rightarrow E^{+} E^{-}$} & $A$ & $0.90798_{+0.00298}^{-0.00402}$ & $0.89357_{+0.00524}^{-0.00376}$ & $0.86080_{+0.00425}^{-0.00328}$ \\
\hline & $B$ & $-5.74818_{-0.02533}^{+0.03299}$ & $-5.46629_{-0.03321}^{+0.03040}$ & $-5.01876_{-0.02570}^{+0.02561}$ \\
\hline & $C$ & $-8.18368_{-0.05541}^{+0.04472}$ & $-6.23857_{-0.02566}^{+0.02602}$ & $-5.19925_{-0.01941}^{+0.01799}$ \\
\hline \multirow{3}{*}{$q \bar{q} \rightarrow Z \rightarrow E^{+} E^{-}$} & $A$ & $0.94114_{+0.00760}^{-0.00508}$ & $0.90624_{+0.00554}^{-0.00414}$ & $0.88538_{+0.00434}^{-0.00339}$ \\
\hline & $B$ & $-3.56798_{-0.04573}^{+0.03728}$ & $-3.22104_{-0.03400}^{+0.03146}$ & $-2.82716_{-0.02557}^{+0.02556}$ \\
\hline & $C$ & $-8.45564_{-0.03792}^{+0.04295}$ & $-6.44243_{-0.02707}^{+0.02677}$ & $-5.28945_{-0.02084}^{+0.01899}$ \\
\hline
\end{tabular}

National Laboratory and University of Chicago Joint Theory Institute (JTI) Grant 0392107-137, and by the U.S. Department of Energy under Grants No. DE-AC02-06CH11357 and No. DE-FG02-90ER40560. Q. H. C. is grateful to C.-P. Yuan for useful discussions. E. L. B. thanks the Galileo Galilei Institute for Theoretical Physics in Florence for hospitality, and the Istituto Nazionale di Fisica Nucleare (INFN) for partial support during the final stages of this work.

Note added: While finalizing the write-up of this work, we became aware of a recent paper [38] in which the $t$ channel production of single $t^{\prime}$ is calculated at the LHC. Our results agree with those in Ref. [38]. 


\section{Appendix A: Standard model parameters}

For our numerical evaluations, we choose the following set of SM input parameters:

$$
\begin{array}{ll}
G_{F}=1.16637 \times 10^{-5} \mathrm{GeV}^{-2}, & \alpha=1 / 137.0359895 \\
m_{Z}=91.1875 \mathrm{GeV}, & \alpha_{s}\left(m_{Z}\right)=0.1186 \\
m_{t}=173.1 \mathrm{GeV}, & \sin ^{2} \theta_{W}^{e f f}=0.2314
\end{array}
$$

Following Ref. [39], we derive the $W$ boson mass as $m_{W}=80.385 \mathrm{GeV}$. Thus, the square of the weak gauge coupling is $g^{2}=4 \sqrt{2} m_{W}^{2} G_{F}$.

\section{Appendix B: Numerical fit of the heavy quark pair production cross section}

In this appendix we use heavy quark pair production via the QCD interaction as a sample illustration of how one may extend the region of applicability of a fit to a wider mass range simply by increasing the number of terms in the polynomial expression. We treat heavy quark production with masses within the range $250 \mathrm{GeV}$ and $700 \mathrm{GeV}$. We choose the following formula to fit the cross section:

$$
\log \left[\frac{\sigma\left(m_{Q}, \mu\right)}{\mathrm{pb}}\right]=\frac{A}{x^{2}}+\frac{B}{x}+C+D x+E x^{2},
$$

where $x=m_{Q} / \mathrm{TeV}$. The fitting functions at the LHC at three energies are given as follows:

$$
\begin{aligned}
& 7 \mathrm{TeV}: 1.33969 x^{2}-10.4918 x+1.36903+1.32168 / x-0.0684983 / x^{2}, \\
& 10 \mathrm{TeV}: 1.87135 x^{2}-9.82576 x+2.34236+1.28886 / x-0.0682711 / x^{2}, \\
& 14 \mathrm{TeV}: 2.16753 x^{2}-9.46830 x+3.33295+1.22263 / x-0.0647251 / x^{2},
\end{aligned}
$$

where the renormalization and factorization scales are chosen as $\mu_{R}=\mu_{F}=\mu_{0}=m_{Q}$. Table $\mathrm{V}$ displays the exact and fitted values of the NLO cross section (pb). The numerical values of the exact and fitted cross sections agree very well.

\section{Appendix C: Influence of parton luminosities on the heavy fermion mass depen-} dence

We comment in this appendix on the origins of the remarkably simple analytic expression Eq. 1 and on its range of applicability in the heavy fermion mass. The expression 
TABLE V: The exact and fitted values of the NLO cross section (pb) for heavy quark pair production at the LHC at three c.m. energies, where the scale is chosen as $\mu_{R}=\mu_{F}=\mu_{0}=m_{Q}$.

\begin{tabular}{c|cc|cc|cc}
\hline$m_{Q}$ & \multicolumn{2}{|c|}{$7 \mathrm{TeV}$} & \multicolumn{2}{c|}{$10 \mathrm{TeV}$} & \multicolumn{2}{c}{$14 \mathrm{TeV}$} \\
$(\mathrm{GeV})$ & Exact & Fit & Exact & Fit & Exact & Fit \\
\hline 250 & 20.51 & 20.50 & 58.34 & 58.32 & 142.10 & 142.06 \\
\hline 300 & 7.289 & 7.291 & 22.21 & 22.21 & 57.03 & 57.04 \\
\hline 350 & 2.940 & 2.939 & 14.38 & 14.38 & 25.78 & 25.78 \\
\hline 400 & 1.301 & 1.301 & 9.565 & 9.561 & 12.74 & 12.74 \\
\hline 450 & 0.617 & 0.618 & 2.285 & 2.286 & 6.742 & 6.743 \\
\hline 500 & 0.310 & 0.310 & 1.224 & 1.224 & 3.771 & 3.770 \\
\hline 550 & 0.162 & 0.162 & 0.685 & 0.685 & 2.203 & 2.204 \\
\hline 600 & 0.088 & 0.088 & 0.398 & 0.398 & 1.336 & 1.337 \\
\hline 650 & 0.049 & 0.049 & 0.239 & 0.239 & 0.837 & 0.837 \\
\hline 700 & 0.028 & 0.028 & 0.147 & 0.147 & 0.539 & 0.539 \\
\hline
\end{tabular}

works well for strong pair production of heavy quarks, as well as for electroweak single heavy fermion production and electroweak pair production of heavy fermions. This apparent process independence suggests that the heavy fermion mass dependence of the parton luminosities [40-42] is playing a dominant role.

The quark-antiquark and gluon-gluon contributions to the cross section for heavy quark pair production at hadron colliders can be written as

$$
\begin{aligned}
& \sigma_{q \bar{q}}(s)=\sum_{q=u, d} \int_{\tau_{m i n}}^{1} d \tau \int_{\tau}^{1} \frac{d x_{a}}{x_{a}}\left[f_{q / P}\left(x_{a}, \mu_{0}\right) f_{\bar{q} / P}\left(\frac{\tau}{x_{a}}, \mu_{0}\right)+(u \leftrightarrow \bar{u})\right] \frac{1}{\hat{s}}\left[\hat{s} \hat{\sigma}_{q \bar{q} \rightarrow Q \bar{Q}}(\hat{s})\right] \\
& \sigma_{g g}(s)=\int_{\tau_{\text {min }}}^{1} d \tau \int_{\tau}^{1} \frac{d x_{a}}{x_{a}} f_{g / P}\left(x_{a}, \mu_{0}\right) f_{g / P}\left(\frac{\tau}{x_{a}}, \mu_{0}\right) \frac{1}{\hat{s}}\left[\hat{s} \hat{\sigma}_{q \bar{q} \rightarrow Q \bar{Q}}(\hat{s})\right]
\end{aligned}
$$

where $\hat{\sigma}$ is the operative parton-level cross section, $\tau_{\min }=4 m_{Q}^{2} / s$, and $\hat{s}=\tau s$ with $s$ being the square of the c.m. energy of LHC. In order to represent a process independent situation, we approximate the (dimensionless) square brackets by one. As a result, the only remaining effect of the heavy quarks appears in the lower limit of integration $\tau_{\min }$. We choose $\mu_{R}=\mu_{F}=m_{Q}$ and use the CTEQ6.6M average PDF set.

Using Eq. C1, we generate "cross sections" as a function of $m_{Q}$, and we then fit the 

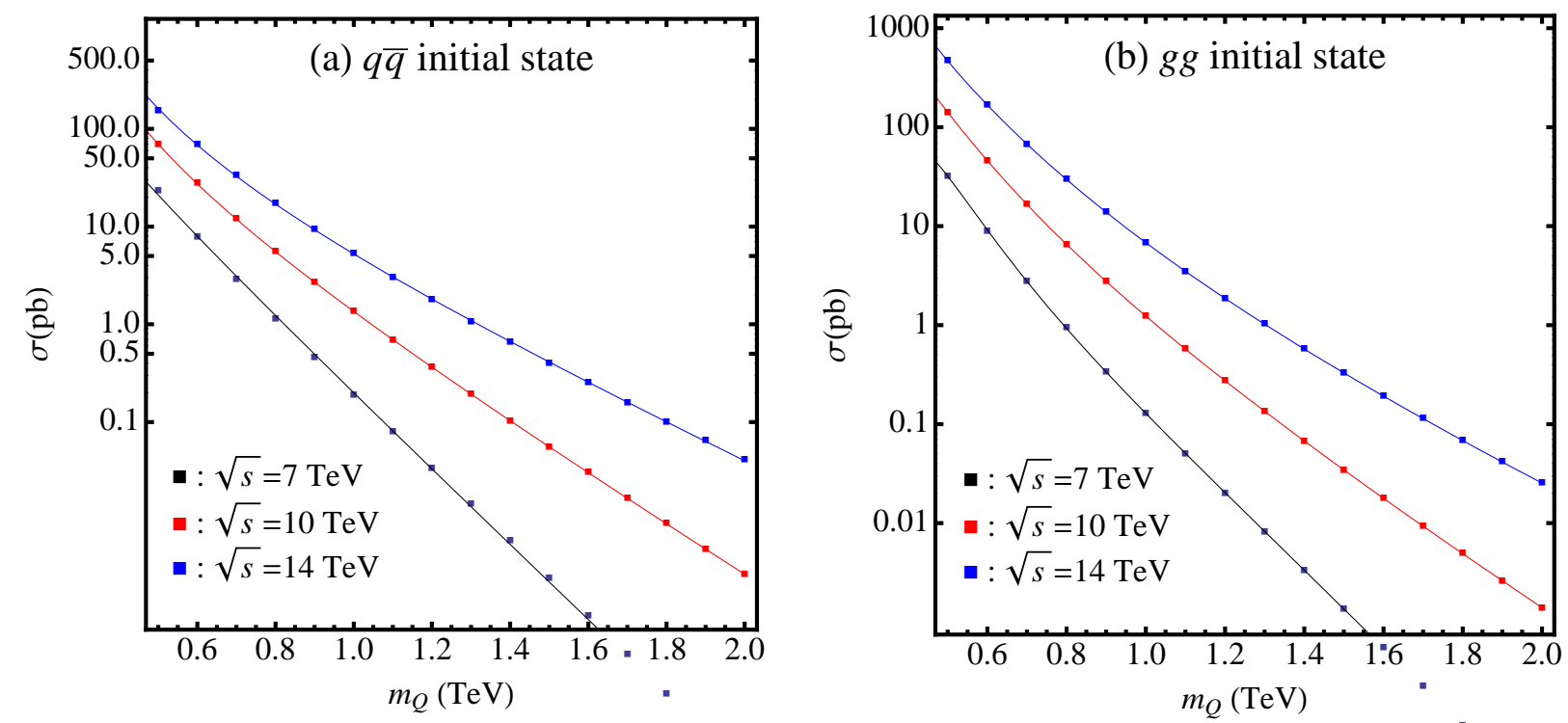

FIG. 9: Fit to the trial scattering cross sections in the range of $0.5<m_{Q}<2.0 \mathrm{TeV}$ : (a) for the quark-antiquark annihilation channel, and (b) for the gluon fusion channel.

results with Eq. 1. The agreement is excellent for the $q \bar{q}$ and $g g$ cases over the range of heavy quark masses shown in Fig. 9, $0.5<m_{Q}<2.0 \mathrm{TeV}$. The square symbols in the figure are the predicted cross sections, and the solid curves represents the fits.

If we try to extend our study to smaller masses than shown in Fig. 9 we find that the simple expression Eq. 1 is no longer adequate, as shown in Fig. 10(a). When $\tau_{\min }$ is too small, we sample parton densities in the region of small Bjorken $x$ where they grow rapidly with decreasing $x$. More terms in the polynomial are needed for a better fit. We can fit the gluon fusion cross section well with the addition of two more terms; see Fig. 10(b) 

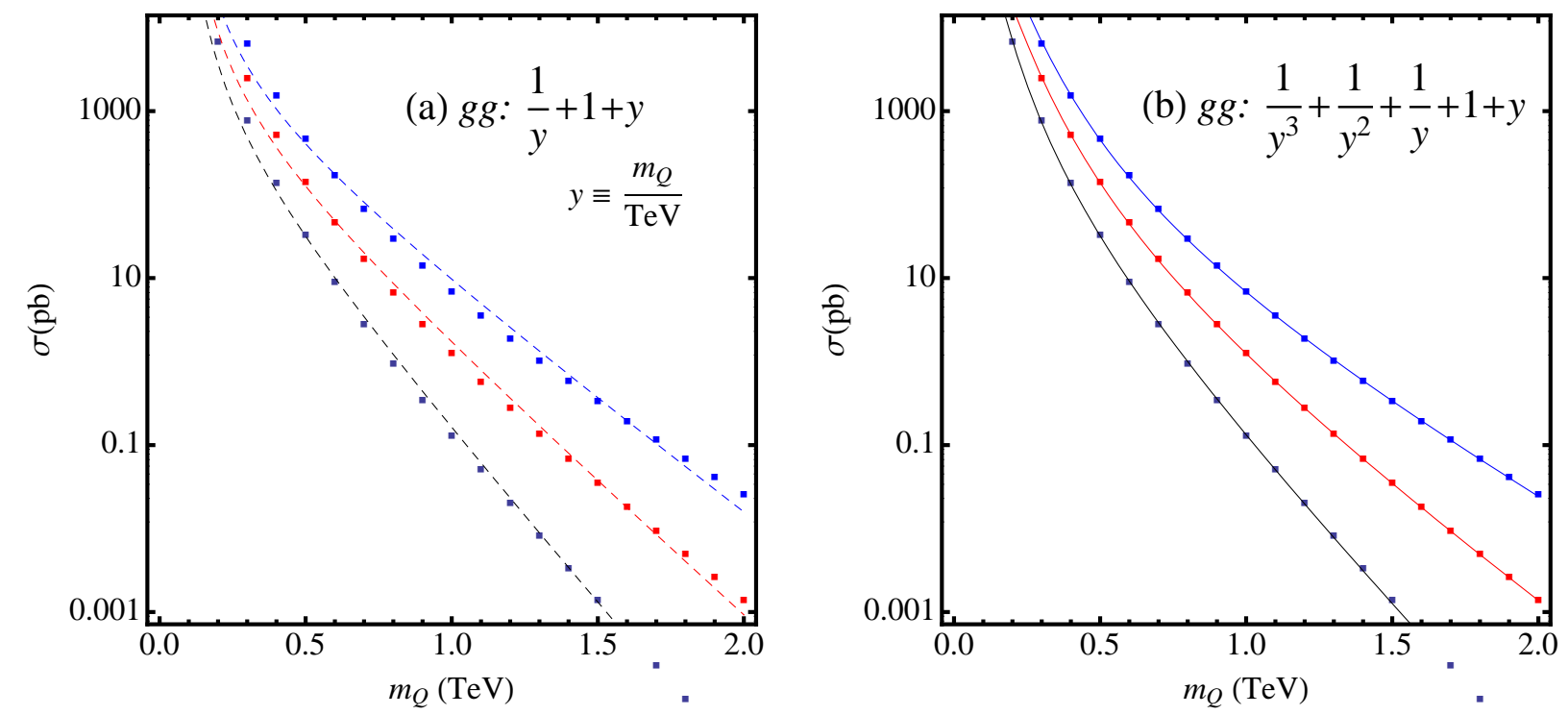

FIG. 10: Fit to the trial scattering cross section for the $g g$ channel extended into the region of small $m_{Q}$.

[1] J. Alwall et al., Eur. Phys. J. C49, 791 (2007), hep-ph/0607115.

[2] G. D. Kribs, T. Plehn, M. Spannowsky, and T. M. P. Tait, Phys. Rev. D76, 075016 (2007), 0706.3718 .

[3] A. Soni, A. K. Alok, A. Giri, R. Mohanta, and S. Nandi (2008), 0807.1971.

[4] M. Bobrowski, A. Lenz, J. Riedl, and J. Rohrwild, Phys. Rev. D79, 113006 (2009), 0902.4883.

[5] M. S. Chanowitz, Phys. Rev. D79, 113008 (2009), 0904.3570.

[6] B. Holdom, W. S. Hou, T. Hurth, M. L. Mangano, S. Sultansoy, and G. Unel (2009), 0904.4698.

[7] A. Soni (2009), 0907.2057.

[8] G. Eilam, B. Melic, and J. Trampetic (2009), 0909.3227.

[9] C. Amsler et al. (Particle Data Group), Phys. Lett. B667, 1 (2008).

[10] F. del Aguila, M. Perez-Victoria, and J. Santiago, JHEP 09, 011 (2000), hep-ph/0007316.

[11] B. A. Dobrescu, K. Kong, and R. Mahbubani, JHEP 06, 001 (2009), 0902.0792.

[12] D. Choudhury, T. M. P. Tait, and C. E. M. Wagner, Phys. Rev. D65, 053002 (2002), hep$\mathrm{ph} / 0109097$. 
[13] N. Arkani-Hamed, A. G. Cohen, E. Katz, and A. E. Nelson, JHEP 07, 034 (2002), hep$\mathrm{ph} / 0206021$.

[14] M. Perelstein, M. E. Peskin, and A. Pierce, Phys. Rev. D69, 075002 (2004), hep-ph/0310039.

[15] T. Han, H. E. Logan, B. McElrath, and L.-T. Wang, Phys. Rev. D67, 095004 (2003), hep$\mathrm{ph} / 0301040$.

[16] H.-C. Cheng, I. Low, and L.-T. Wang, Phys. Rev. D74, 055001 (2006), hep-ph/0510225.

[17] F. del Aguila, L. Ametller, G. L. Kane, and J. Vidal, Nucl. Phys. B334, 1 (1990).

[18] J. A. Aguilar-Saavedra, Phys. Lett. B625, 234 (2005), hep-ph/0506187.

[19] S. Matsumoto, M. M. Nojiri, and D. Nomura, Phys. Rev. D75, 055006 (2007), hep$\mathrm{ph} / 0612249$.

[20] A. Freitas and D. Wyler, JHEP 11, 061 (2006), hep-ph/0609103.

[21] P. Meade and M. Reece, Phys. Rev. D74, 015010 (2006), hep-ph/0601124.

[22] Q.-H. Cao, C. S. Li, and C. P. Yuan, Phys. Lett. B668, 24 (2008), hep-ph/0612243.

[23] A. Belyaev, C.-R. Chen, K. Tobe, and C. P. Yuan, Phys. Rev. D74, 115020 (2006), hep$\mathrm{ph} / 0609179$.

[24] R. Contino and G. Servant, JHEP 06, 026 (2008), 0801.1679.

[25] J. A. Aguilar-Saavedra (2009), 0907.3155.

[26] P. Nason, S. Dawson, and R. K. Ellis, Nucl. Phys. B303, 607 (1988).

[27] W. Beenakker, H. Kuijf, W. L. van Neerven, and J. Smith, Phys. Rev. D40, 54 (1989).

[28] P. Nason, S. Dawson, and R. K. Ellis, Nucl. Phys. B327, 49 (1989).

[29] W. Beenakker, W. L. van Neerven, R. Meng, G. A. Schuler, and J. Smith, Nucl. Phys. B351, 507 (1991).

[30] D. Stump et al., JHEP 10, 046 (2003), hep-ph/0303013.

[31] A. D. Martin, W. J. Stirling, R. S. Thorne, and G. Watt, Eur. Phys. J. C63, 189 (2009), 0901.0002.

[32] R. D. Ball et al. (NNPDF), Nucl. Phys. B809, 1 (2009), 0808.1231.

[33] N. Kidonakis and R. Vogt, Phys. Rev. D78, 074005 (2008), 0805.3844.

[34] T. Stelzer, Z. Sullivan, and S. Willenbrock, Phys. Rev. D56, 5919 (1997), hep-ph/9705398.

[35] F. del Aguila and J. A. Aguilar-Saavedra, Nucl. Phys. B813, 22 (2009), 0808.2468.

[36] J. A. Aguilar-Saavedra (2009), 0905.2221.

[37] F. del Aguila and J. A. Aguilar-Saavedra, Phys. Lett. B672, 158 (2009), 0809.2096. 
[38] J. M. Campbell, R. Frederix, F. Maltoni, and F. Tramontano (2009), 0907.3933.

[39] G. Degrassi, P. Gambino, M. Passera, and A. Sirlin, Phys. Lett. B418, 209 (1998), hep$\mathrm{ph} / 9708311$.

[40] E. Eichten, I. Hinchliffe, K. D. Lane, and C. Quigg, Rev. Mod. Phys. 56, 579 (1984).

[41] R. K. Ellis, W. J. Stirling, and B. R. Webber, QCD and Collider Physics (Cambridge Monographs on Particle Physics, Nuclear Physics and Cosmology) (Cambridge University Press, 2003).

[42] C. Quigg (2009), 0908.3660. 